\title{
Exploration of the Possibility of Ionic Polyurethane used as IPMC Substrate
}

\author{
Yanou $\mathrm{Hu}^{1}$, Huiqin $\mathrm{Lian}^{1, *}$, Yueting $\mathrm{Li}^{1,2}$, Wei Chang ${ }^{1,2}$, \\ ${ }^{1}$ Beijing Key Lab of Special Elastomer Composite Materials, College of Materials Science and Engineering, Beijing Institute of \\ Petrochemical Technology, Beijing 102617, China. \\ ${ }^{2}$ College of Materials Science and Engineering, Beijing University of Chemical Technology, Beijing 100029, China.
}

\begin{abstract}
In this study, polyurethane elastomer containing sulfonic acid based was synthesized by two steps. Poly(tetramethylene ether) glycol with $2000 \mathrm{~g} / \mathrm{mol}$ number average molecular weight were used as soft segment, 4,4-methylenebis(phenyl isocyanate), and 1,2-dihydroxy-3-propanesulfonic acid salt were used to compose the segment of the polyurethane elastomer. We report for the first time the fabrication of the ionic polyurethane actuators with graphene as electrodes. Characterize the image and structure of the materials by Fourier Transform infrared(FT-IR), X-ray diffraction(XRD) and scanning electron microscopy (SEM), results showed that this materials prepared certain performance of electromechanical response.
\end{abstract}

\section{Introduction}

Electroactive polymer, ionic polymer-metal composite (IPMC), has attracted much attention as a good candidate for robotic actuators, artificial muscle, and dynamic sensors because of its outstanding advantages including flexibility, lighter weight, biocompatibility, and a large displacement under low potential(1-5 V) stimuli $[1,2]$. It was considered as a good candidate for robotic actuators, artificial muscle, and dynamic sensors due to its outstanding advantages including flexibility, low power consumption, high energy density, and a large displacement under low voltage[3]. Typically, an IPMC actuator composed of an ion-exchanging polymer film sandwiched with two noble metal electrodes had attracted much attention. In general, a typical IPMC consists of a thin ion-exchange polymer membrane covered with a thin metal layer from both sides, and the metal layers serve as surface electrodes[4]. Its electromechanical mechanism is that hydrated metal cations inside the actuator migrate toward cathode under electrical field, which causes the large deformation, and thus strong blocking force and displacement are produced. However, the existing IPMC actuators have two significant drawbacks, low generative blocking force and short nonwater working time, both of which are closely related to the solvent losing in the polymer because of the evaporation, electrolysis, and the low

mechanical property of polymer[5]. So we do this work to explore the possibility of ionic polyurethane used as IPMC substrate.

\section{Experimental Section}

2.1 Materials and method. 3-Chloro-2-hydroxypropanesulfonic acid sodium salt (CHPS-Na), Polytetramethylene ether glycol (PTMG, $\mathrm{Mn}=2000 \mathrm{~g} / \mathrm{mol}$ ), 4,4'-diphenylmethane diisocyanate (MDI), Tetra-n-butylammonium bromide (TBAB) and HI acid $(55 \mathrm{wt} \%)$ were purchased from Aladdin-reagent
Inc. .PTMG was dried under vacuum at $100^{\circ} \mathrm{C}$ for $2 \mathrm{~h}$ before use. Other chemicals were used as received. DHPA is synthesized via substitution reaction of CHPS-Na using TBAB as catalyst[6]. Polyurethane elastomer containing sulfonic acid based was synthesized by two steps. Poly(tetramethylene ether) glycol with $2000 \mathrm{~g} / \mathrm{mol}$ number average molecular weight were used as soft segment, 4,4-methylenebis(phenyl isocyanate), and 1,2-dihydroxy-3-propanesulfonic acid salt were used to compose the segment of the polyurethane elastomer[7-9]. The rout of synthesis a typical PU-S though a polyaddition reaction of polyol, isocyanate and chain extender is shown in Fig.1. And Series of PU-S are synthesized and the formulations are listed in Table 1 .

GO was prepared from natural graphiteflake using the modified Hummers method[10]. IPMC actuators is prepared into a sandwich structure, outer are GO and the middle tier is ion polyurethane. Then the GO layer was hung over a $\mathrm{HI}$ acid solution in a sealed beaker at $80{ }^{\circ} \mathrm{C}$ to transfer both up and bottom surfaces to rGO by in situ reduction for a certain time[11, 12].

Characterizations. The PU was characterized by FT-IR analysis in a Nicolet 6700 spectrometer in the range of 400-4000 cm-1. X-ray diffraction (BRUKER D8) patterns were obtained on a Bruker D8 diffractometer equipped with $\mathrm{Cu} \mathrm{K}$ source.. The morphology of films

\begin{tabular}{|c|c|c|c|c|c|c|}
\hline Samples & $\begin{array}{l}\text { PTME- } \\
2000 \\
(\mathrm{~mol})\end{array}$ & $\begin{array}{l}\text { BDO } \\
(\mathrm{mol})\end{array}$ & $\begin{array}{l}\mathrm{DH} \\
\mathrm{PA} \\
\text { (mo } \\
\text { 1) }\end{array}$ & $\begin{array}{l}\text { MDI } \\
(\mathrm{mol})\end{array}$ & $\begin{array}{l}\text { Hard } \\
\text { conte } \\
\text { nt } \\
(\% \\
(\%\end{array}$ & $\begin{array}{l}\text { Sulfonic } \\
\text { group } \\
\text { content } \\
(\mathrm{mmol} / \mathrm{g})\end{array}$ \\
\hline PU-30 & 0.1 & 0.18 & 0 & 0.28 & 30 & 0 \\
\hline PU-S30 & 0.1 & 0 & 0.14 & 0.24 & 30 & 0.50 \\
\hline PU-S50 & 0.1 & 0 & 0.41 & 0.51 & 50 & 1.02 \\
\hline PU-S100 & 0 & 0 & 0.24 & 0.24 & 100 & 2.33 \\
\hline
\end{tabular}

was examined using scanning

Table 1. Formulations of PU-S samples 


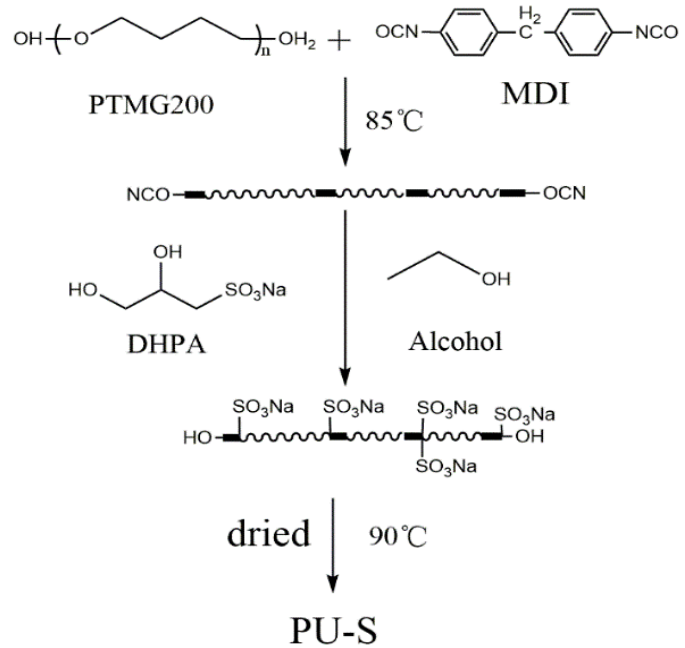

Figure 1. Schematic representation for the synthesis of PU-S.

electron microscopy (SEM, COXEM EM-20). The actuation performance was carried out using a homemade setup equipped with an electric balance. All the samples were cut into $3095 \mathrm{~mm} 2$ rectangles, including $5 \mathrm{~mm}$ of electrode contact area. One end of the SRGO film was fixed between two $\mathrm{Cu}$ electrodes. All measurements were carried out in air at room temperature.

\section{Results and discussion}

\subsection{FT-IR spectra}

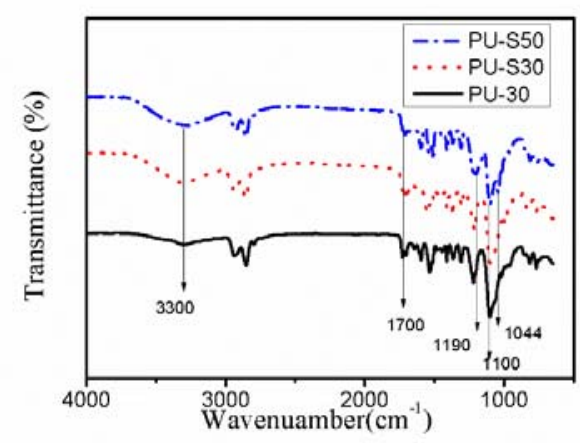

Figure 2. FT-IR spectra patterns of the Pus

The FTIR spectra for PU-S samples are shown in Figure 2. From the FT-IR spectrum of PU-30, the broad band at $3300 \mathrm{~cm}-1$ is attributed to the N-H stretching vibration. The bands centered at $1700 \mathrm{~cm}-1$ and $1100 \mathrm{~cm}-1$ are due to the $\mathrm{C}=\mathrm{O}$ and $\mathrm{C}-\mathrm{O}-\mathrm{C}$, respectively. The disappearance of characteristic peaks of $\mathrm{N}=\mathrm{C}=\mathrm{O}$ at $2241 \mathrm{~cm}-1$ indicated the formation of PU. Comparing with that of PU-30, the spectra of PU-S30 and PU-S50 show the band at 1210 $\mathrm{cm}-1$ and $1044 \mathrm{~cm}-1$ due to the -SO3-group[26]. It is noticed that with the content of SO3- increase, the relative intensity of peaks at $1210 \mathrm{~cm}-1$ and $1044 \mathrm{~cm}-1$ increase. Therefore, series of PUs containing sulfonic group are synthesized successfully.

\subsection{XRD characterization}

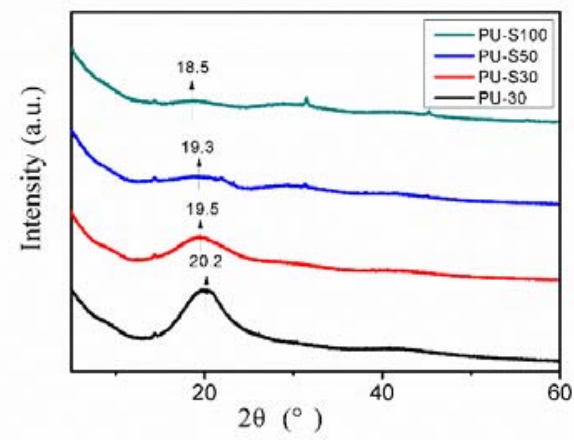

Figure 3. XRD patterns of the synthesized Pus

The The XRD patterns of IPU films are shown in Fig.3. All films show a broad peak centered around $2 \theta=19^{\circ}$ due to polyol[25]. The broad peak $20.2^{\circ}$ in PU-30, $19.5^{\circ}$ in PU-S30, $19.3^{\circ}$ in PU-S50 and $18.5^{\circ}$ in PU-S100, characteristics of the crystallization of the soft segments this is because there are large amounts of amorphous or microcrystalline in the system. Along with the increase of hard segment content, the diffuse peak gradually reduced, and two sharp peak arise in the PU samples with the hard segment content is $50 \%$ and $100 \%$. This suggests that the crystalline region appeared in the PU. An elevated content of the hard phase created more physical cross-links and second bonds, which resulted in reducing the flexibility of soft segments and limiting their overall backbone mobility. Polyurethanes with crystalline structures can improve its mechanical properties than those with amorphous segments, which contributes to the aim of this work. So we chose the PU samples with the hard segment content is $50 \%$ as the base material of IPMC.

\subsection{Ion-exchange Capacity}

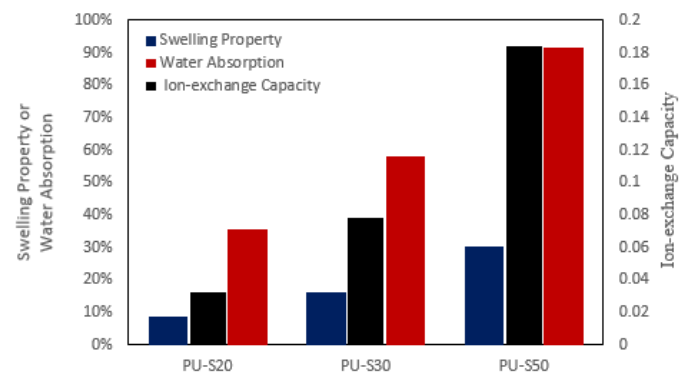

Figure 4. Swelling Property, Water Absorption and Ion-exchange Capacity of the Pus

The swelling property, water absorption and ion-exchange capacity of the PUs was shown in the Fig.4 Obviously, the swelling property, water absorption and ion-exchange capacity are all improve along with the content of Sulfonic group. This portends that the PU-S we synthesized can be used as IPMC material. 


\subsection{SEM observation}

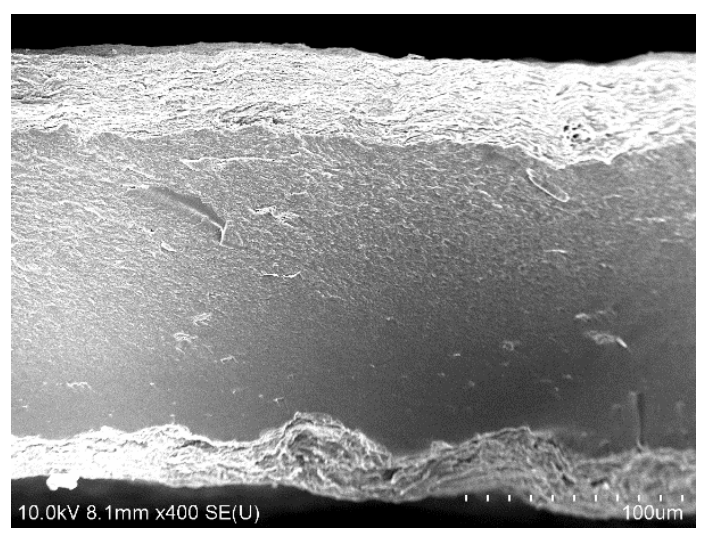

Figure 5. SEM images of IPMC based on the PU.

The figure 5 shows that the PU membrane $r$ is about $100 \mu \mathrm{m}$ thick, on both sides of the membrane is the electrode which aer about $20 \mu \mathrm{m}$.Moreover, the PU membrane close combined with graphene electrodes, which has a good electrical conductivity.

\subsection{Actuation and blocking force}

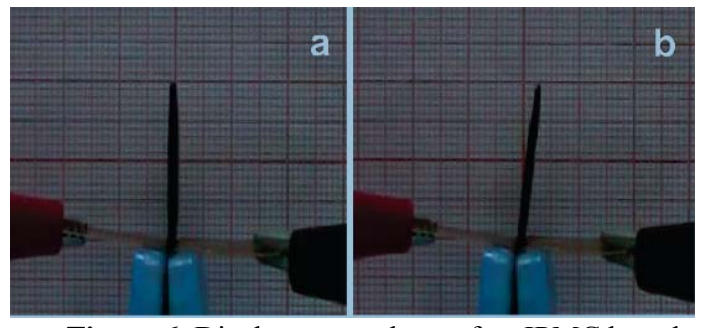

Figure 6. Displacement photo of an IPMC based on the PU membrane (a) before test and (b) driven under a $3 \mathrm{~V}$ electrical filed.

The actuator performance of the as-prepared PU membranes was measured by a cantilevered actuation system. The deflection of the membrane was tested by fixing one end of the membrane in the apparatus and measuring the movement of the free end. Figure $6 \mathrm{a}, \mathrm{c}$ show the photos of PU-based IPMC actuators with a piece of squared paper as background before test, which is upright. When a voltage of $3 \mathrm{~V}$ was used to drive the IPMC actuators, IPMC exhibits a perceptibly displacement (Figure 6b).

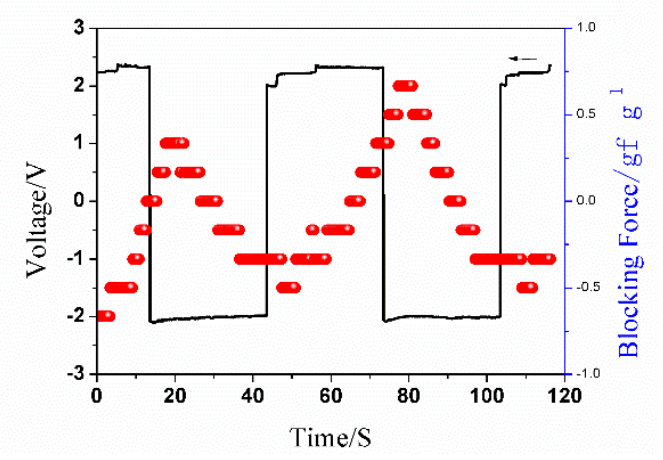

Figure 7. Blocking force the actuator
We prepared the IPMC actuator by using PU-S50 as matrix, and graphene as electrode. The material has a certain actuating performance and its braking force is $0.75 \mathrm{gf} / \mathrm{g}$, which compared with the Nafion-based IPMC material's $10 \mathrm{gf} / \mathrm{g}$ braking force, there also is a large gap. We will focused on how to enhance its actuation performance in the further research.

\section{Conclusions}

In this study, we successfully synthesized ionic polyurethane which can be proved by FT-IR spectra pattern and XRD analysis. And then, we prepared the ionic polyurethane actuators with graphene as electrodes. The PU-based IPMC material prepared certain performance of electromechanical response.

\section{Acknowledgements}

This work was supported by the Graduate student innovation project (NO.15033981002/086), the Natural Science Foundation of China (NSFC, Nos. 51063009, 21271031, 51203012), the Beijing Natural Science Foundation of China (Nos. 2132009, 2122015), undergraduate Research Training Program of Beijing (2015J00002) and the project about the promotion of innovation in Beijing universities funded by Beijing Municipal commission of education of China.

\section{References}

[1] Lian H, Qian W, Estevez L, Liu H, Liu Y, Jiang T, Wang K, Guo W, and Giannelis EP. Sensors and Actuators B: Chemical 2011;156(1):187-193.

[2] Shahinpoor M. Electrochimica Acta 2003;48(14-16):2343-2353.

[3] Lee DY, Park I-S, Lee M-H, Kim KJ, and Heo S. Sens. Actuators, A 2007;133(1):117-127.

[4] Akle BJ, Bennett MD, and Leo DJ. Sensors and Actuators A: Physical 2006;126(1):173-181.

[5] Burgaz E, Lian H, Alonso RH, Estevez L, Kelarakis A, and Giannelis EP. Polymer 2009;50(11):2384-2392.

[6] Barrioni BR, de Carvalho SM, Oréfice RL, de Oliveira AAR, and Pereira MdM. Materials Science and Engineering: C 2015;52(0):22-30.

[7] Zhang F-x and Wei X-l. Zhongguo Jiaonianji 2009;18(6):12-14.

[8] Zhang F-X, Wei X-1, and Zhang C. Zhongguo Jiaonianji 2008;17(11):9-12.

[9] Rafiemanzelat F, Fathollahi Zonouz A, and Emtiazi G. Polymer Degradation and Stability 2012;97(1):72-80.

[10] Hummers WS and Offeman RE. Journal of the American Chemical Society 1958;80(6):1339-1339.

[11] Hu Y, Lian H, Zu L, Jiang Y, Hu Z, Li Y, Shen S, Cui X, and Liu Y. J. Mater. Sci. 2015:Ahead of Print. 
[12] Pei S, Zhao J, Du J, Ren W, and Cheng H-M. Carbon 2010;48(15):4466-4474. 